\title{
UNA APROXIMACIÓN A LOS ESCRITOS DE CARÁCTER CIENTÍFICO DE GOETHE
}

\author{
Catalina Soto de Prado y Otero \\ Universidad de Valladolid \\ catalina@fyl.uva.es
}

\section{RESUMEN}

Nos gustaría con este artículo acercarnos a los estudios de Goethe sobre ciencias naturales, y más concretamente a destacadas ediciones que han recogido esta parte de su obra, especialmente la edición leopoldina. Es también objeto de este artículo valorar la repercusión que ha tenido la obra científica de Goethe en el ámbito hispanohablante y proponer unas ediciones concretas a la hora de trabajar los contenidos de carácter científico de Goethe.

Palabras Clave: Goethe, obras científicas, Academia leopoldina, biblioteca de Goethe, recepción.

\section{ABSTRACT}

Through this article, we would like to approach Goethe`s studies on natural sciences and, more specifically, several prominent editions that have gathered this aspect of his work, specially the Leopoldina edition. This article also intends to value the repercussion that Goethe's scientific work has had in the spanish-speaking world and to propose a number of concrete editions in order to deal with those contents of a scientific character in Goethe`s work.

KEY WORDS: Goethe, scientific works, Leopoldina Academy, Goethe`s library, reception. 


\section{INTERESES CIENTÍFICOS DE GOETHE}

Nos gustaría con este artículo acercarnos a los estudios de Goethe sobre ciencias naturales, y más concretamente a destacadas ediciones que han recogido esta parte de su obra, especialmente la edición Leopoldina. Es también objeto de este artículo valorar la repercusión que ha tenido la obra científica de Goethe en el ámbito hispanohablante y proponer unas ediciones concretas a la hora de trabajar los contenidos de carácter científico $^{1}$ de Goethe. Hay que señalar que cuando nos referimos a los «estudios» de Goethe estamos hablando de sus investigaciones científico-naturales junto con sus primeras conclusiones, combinaciones e hipótesis. Goethe empezó con estos estudios en el año 1780 , en un primer momento adquiriendo los conocimientos previos en anatomía y mineralogía según el estado de la ciencia en ese momento, después -con gran empeño- en la botánica siguiendo las premisas de Linneo. Así comenzaba una verdadera transformación en el comportamiento del poeta hacia la naturaleza, extinguiéndose un periodo en el que a Goethe no sólo le bastaba para hablar sobre la naturaleza el conocimiento procedente de los sentidos. Los intereses científico-naturales de Goethe eran muy diversos y se centraban casi siempre en cuestiones prácticas, así como en las diferentes necesidades que surgían en su trabajo al servicio del Ducado de Sajonia-Weimar-Eisenach. De este modo, se entiende por ejemplo la reanudación de la minería en Ilmenau -localidad a unos $50 \mathrm{Km}$ de Weimar en el bosque de Turingia- y las investigaciones que para ello llevó personalmente Goethe en las que se encuentran los verdaderos orígenes de sus intereses geológicos y minerales, como hemos explicado extensamente en la obra Goethe y el bosque a través de sus textos (cf. Soto de Prado y Otero, 2009). En una de sus tantas charlas con su amigo el Canciller von Müller $^{2}$ y Friedrich Soret ${ }^{3}$-que recoge Eckermann en sus Conversaciones ${ }^{4}$-, le escribe el 16 de marzo de 1824:

Ich kam höchst unwissend in allen Naturstudien nach Weimar, und erst das Bedürfnis, dem Herzog bei seiner mancherlei Unternehmungen, Bauten, Anlagen, praktische Ratschläge geben zu können, trieb mich zum Studium der Natur. Ilmenau hat mich viel Mühe und Geld gekostet, dafür habe ich auch etwas dabei gelernt und mir eine Anschauung der Natur erworben, die ich unter keinen Preis umtauschen möchte. ${ }^{5}$

\footnotetext{
${ }^{1}$ No economizamos en la terminología al referirnos al término «científico-natural» en relación a las ciencias de la naturaleza. Sin embargo, en muchos puntos de este artículo por razones de «economía del lenguaje» abreviaremos calificando estos estudios de Goethe tan sólo como «científicos», sin dejar de considerar que las otras investigaciones de Goethe de carácter jurídico, literario o social revestían también carácter científico aunque se tratara de ciencias humanas.

${ }^{2}$ Friedrich Theodor Adam Heinrich von Müller (1779-1849). Jurista y hombre de Estado. Cabe destacar en su dilatada carrera profesional su nombramiento como canciller de Weimar en 1815 y en 1829 miembro del Geheimrat o Consejo Secreto. Fue el albacea del testamento de Goethe.

${ }^{3}$ Frédéric Jean Soret (1795-1865). Teólogo ginebrino, científico y escritor. Entre 1822 y 1836 fue el tutor en Weimar del príncipe heredero Karl Alexander.

${ }^{4}$ Goethe: Gespräche 1824, S. 64. Digitale Bibliothek Band 10: Goethe: Briefe, Tagebücher, Gespräche, S. 30140 (vgl. Goethe-Gespr. Bd. 5, S. 50-51).

${ }^{5}$ Esta cita la hemos traducido así: «Llegué a Weimar con un alto grado de ignorancia en todos los estudios sobre ciencias naturales y tan sólo el deber de poder dar consejos prácticos al duque en las distintas iniciativas, edificaciones y parques me llevó al estudio de la naturaleza. Ilmenau nos ha costado mucho esfuerzo y dinero pero a la vez he aprendido algo y alcanzado un concepto de la naturaleza que no cambiaría por nada del mundo.
} 
Goethe dirigió su atención a una gama de fenómenos naturales extraordinariamente amplia. La Mineralogía, la Geología y la Botánica se convirtieron en disciplinas objeto de intenso estudio cuando en plena juventud se instaló en Weimar por invitación del Duque Carl August, ya que sus obligaciones oficiales incluían la inspección de minas, las frecuentes visitas a los invernaderos en Belvedere y a los jardines botánicos, y su residencia oficial era un pabellón situado en un parque a las afueras de la ciudad, concretamente en el Ilmpark. Es típico de la personalidad de Goethe que esas circunstancias de su vida en Weimar despertaran en él una fascinación tanto por los minerales como por las plantas. Su interés por las plantas se vio estimulado además por su toma de contacto con los cercanos bosques de Turingia, así como por los herbolarios que conoció en la campiña silvestre. De hecho, en 1776 comienza una colección de grabados sobre paisajes, plantas y motivos naturales y en 1785 organiza en el jardín de su casa en la Frauenplatz de Weimar el botanisches Beet o bancal botánico para poder estudiar el crecimiento de las plantas. Asimismo, destacó como investigador de los fenómenos de la percepción cromática y óptica, materia que le interesaría durante el resto de su vida y que expuso en su Teoría de los colores, obra de la que Goethe se sentía enormemente orgulloso. Esta obra se publicaría con integridad en 1810. Cuando se presta atención a los escritos científicos de Goethe se nos revela una faceta poco conocida de este autor. Como explica Diego Sánchez Meca en el estudio preliminar (1997: XI), lo que se nos presenta no es el rostro del insigne poeta y dramaturgo

(...) capaz de suscitar tanta admiración y entusiasmo como para poder ejercer un indiscutido reinado literario que dura ya dos siglos, sino el de un hombre obsesionado por penetrar en el secreto del «orden móvil» de la vida, de la síntesis viviente de necesidad y libertad, que busca sin descanso el modelo teórico capaz de captar las regularidades ideales en el devenir temporal de las metamorfosis de las formas.

Aunque Goethe haya pasado a la historia como uno de los grandes genios de la literatura universal y sea poco conocido en el ámbito de las ciencias naturales, lo cierto es que Goethe dedicó a la observación y a la experimentación científica de los procesos naturales tanto tiempo y esfuerzo como a la literatura. De hecho, Goethe comenzó sus investigaciones como naturalista desde muy joven y las continuó hasta su muerte como atestigua la carta de su secretario, Eckermann ${ }^{6}$, a Kiesewetter ${ }^{7}$ del 3 de abril de 1832 en la que además de comunicarle el fallecimiento del poeta el 22 de marzo de ese mismo año, también le comunica lo siguiente:

Después de que, el último verano, Goethe acabara la segunda parte de su inmortal Fausto, el invierno pasado se ocupó preferentemente de estudios sobre ciencia natural. Tomó parte

${ }^{6}$ Johann Peter Eckermann (1792-1854). Eckermann es recordado principalmente por sus contribuciones al conocimiento sobre el gran poeta, gracias a su obra Conversaciones con Goethe (1836-1848). Goethe confió a Eckermann la publicación de sus Nachgelassene Schriften (obras póstumas) (1832-1833). También fue coeditor junto con Friedrich Wilhelm Riemer (1774-1845) de las ediciones completas de las obras de Goethe en 40 tomos (1839-1840).

${ }^{7}$ Raffael Georg Kiesewetter (1773-1850), Edler von Wiesenbrunn, jurista austriaco y musicólogo. 
en la discusión parisina entre Cuvier y Saint-Hilaire, y escribió un importante ensayo sobre temas osteológicos y sobre el problema del análisis y la síntesis de las ciencias naturales en general ${ }^{8}$

Para comprender mejor esta amplísima faceta de Goethe como científico es muy revelador entrar en su biblioteca personal, ya que ésta da buena cuenta de sus intereses personales.

\section{LA BIBLIOTECA PERSONAL DE GOETHE}

La biblioteca personal de Goethe se encontraba en su casa de la Frauenplan en Weimar y hoy en día se conserva casi íntegramente; este lugar alberga en la actualidad el Goethe-Nationalmuseum y es uno de los centros más importantes dedicados a Goethe y su estudio. En esta biblioteca se encuentran 5424 títulos que corresponden a alrededor de 7500 volúmenes y llenan baldas, estanterías, armarios y atriles. Goethe se sentía muy a gusto en las bibliotecas y hay que añadir que todo el fondo bibliográfico que tenía no era en aras de la ostentación sino por motivos de trabajo. Podemos intuir la disposición en la que estuvieron ordenados los libros, pues aún se conservan algunos rótulos en las estanterías de la biblioteca de cuando Kräuter ${ }^{9}$, durante unos años secretario de Goethe, los iba clasificando. Sin embargo, durante la II Guerra Mundial se tuvo que vaciar la biblioteca y otras muchas estancias de esta casa, no pudiéndose posteriormente reconstruir del todo su orden original. Hoy día los volúmenes se encuentran clasificados por áreas científicas, numerados de forma continua y catalogados por Hans Ruppert como explicaremos más adelante. En líneas generales podemos clasificar el fondo bibliográfico de la siguiente manera: un cuarto del total trata sobre lingüística y literatura. Unas $1150^{10}$ obras son de carácter puramente científico, lo que indica que la ciencia para Goethe no era un hobby o mera afición; también hay muchos volúmenes muy valiosos sobre arte, así como obras sobre derecho, política y economía. Además, también se encuentran más de cien periódicos y revistas nacionales y extranjeros que durante muchos años fue comprando Goethe. Los diccionarios y enciclopedias los usaba Goethe con mucha frecuencia teniéndolos siempre a mano en su cuarto de trabajo. También hay obras de literatura universal desde Suecia hasta La India, tanto en versiones originales como traducidas. Paradójicamente, las ediciones de sus propias obras no se encuentran íntegramente en su biblioteca. Muchos de sus libros llevan su ex libris y múltiples dedicatorias e inscripciones que documentan la amistad y relación de Goethe con autores, editores, traductores y científicos de todo el mundo. Actualmente ya no está abierta al público; tan sólo se puede divisar desde un cuarto previo a su despacho. No obstante, los investigadores y científicos con previa autorización sí que tenemos la posibilidad de consultar estos pre-

\footnotetext{
${ }^{8}$ Carta citada por Sánchez Meca (1997).

${ }^{9}$ Friedrich Theodor Kräuter (1790-1856), secretario y bibliotecario de la Biblioteca Anna Amalia de Weimar.

${ }^{10}$ Concretamente, y siguiendo la catalogación de Ruppert, confirman este dato los 1139 títulos de obras de ciencias naturales y medicina, 35 títulos de obras de matemáticas, 40 títulos de obras sobre balneoterapia, 35 títulos correspondientes a obras sobre economía forestal y agricultura y 35 sobre técnica.
} 
ciosos ejemplares que hoy día custodia la Stiftung Weimarer Klassik, fundación que vela por todo el legado de Goethe.

Hemos creído importante en este artículo explicar brevemente cómo se llevó a cabo la catalogación de la biblioteca de Goethe. Esta biblioteca fue catalogada en varias ocasiones (cf. Maria Wagner, 2007); la primera ocasión en la que encontramos constancia de ello data del año 1788 y se trata de un listado o guía con el título de «Verzeichnis derjenigen Bücher, welche sich in der Bibliothek Ihro des Herrn Geheimden Rath von Goethe Hochwohlgeb. Vorfinden. $1788 »^{11}$; se piensa que es obra de Christian Georg Vogel y recopila -según uso y costumbre de la época- las obras de consulta que se encuentran en la biblioteca de Goethe ordenadas según formatos, recogiendo tan sólo 317 ejemplares; además, en este listado aparecen para su catalogación muchas abreviaturas y algunas faltas de trascripción. Más conocido y mucho más extenso, pero realmente intrincado para los requisitos actuales, es el «Catalogus bibliotheca Goethianae» de Theodor Kräuter y que llevó hasta el fallecimiento de Goethe. También es conocida la catalogación de Carl Ruhland (1868-1907), quien estableció listas de los libros que figuraban en la biblioteca de Goethe clasificados en 17 materias diferentes. Otro autor que trabajó arduamente en catalogar de forma novedosa y bien articulada el fondo bibliográfico de Goethe fue Carl Schüddekopf (1861-1917) pero desgraciadamente este trabajo se interrumpió con su muerte. Posteriormente, en concreto desde 1953, un equipo de científicos trabajó bajo la dirección del Dr. Hans Ruppert en la elaboración de un catálogo completo de las obras que componían la biblioteca de Goethe presentándose públicamente en el año 1958 (cf. Ruppert, 1958). Hoy en día y con el objeto de unificar criterios a la hora de trabajar con la biblioteca de Goethe se toma la numeración propuesta por Ruppert.

\section{LAS EDICIONES DE LA OBRA DE CARÁCTER CIENTÍFICO DE GOETHE}

Las obras científicas de Goethe más significativas se encuentran dentro de ediciones que comprenden sus obras completas; por este motivo, al tratarse de un autor tan relevante, existen muchas editoriales y editores que a lo largo de la historia han publicado sus obras o parte de ésta; hablamos por supuesto de ediciones en lengua alemana. Sin embargo, por las características de nuestro estudio, señalaremos solamente aquellas ediciones donde la aparición de las obras de carácter científico tenga cierta relevancia:

1. Naturwissenschaftliche Schriften, II parte de la Goethes Werke o edición de Weimar:

Las obras de carácter científico-natural se publicaron en su mayor parte en la famosa «Edición de Weimar». Son las llamadas Naturwissenschaftliche Schriften de la Goethes Werke u obras de Goethe. Esta edición fue encargada por la Gran Duquesa Sophie von Sachsen; por este motivo a la Weimarer Ausgabe se la conoce también como Sophie Ausgabe. Esta edición reúne 133 volúmenes que se reparten en 4 secciones: Werke (obras), Naturwissenschaftliche Schriften (escritos de carácter científico), Tagebücher (diarios) y

\footnotetext{
${ }^{11}$ «Listado de aquellos libros que se encuentran en la biblioteca del consejero secreto von Goethe».
} 
Briefe (cartas). Lo editó Böhlau en Weimar entre 1887 y 1919. Esta edición general de las obras de Goethe reproduce, a excepción de los documentos administrativos, todos los textos de Goethe, incluso los menores fragmentos y las más pequeñas variaciones. La sección II -que como hemos dicho comprende los escritos de carácter científico- se organiza como sigue: Farbenlehre (vols. 1-4), Chromatik y Paralipomena del volumen 5 (vols. 5.1. y 5.2.), Morphologie (vols. 6-8), Mineralogie und Geologie (vols. 9-10), Allgemeine Naturlehre (vol. 11-12), Nachträge zu Band 6-12 (vol.13) ${ }^{12}$.

En esta gran edición de Weimar, los volúmenes que versan sobre ciencias naturales comprenden 13 del total. Y como apunta Sánchez Meca (1997: XI):

Si se calcula además que, en los cincuenta volúmenes de cartas y en los treinta y siete de diarios, muchas de sus páginas tratan temas de ciencias naturales, esta simple cuantificación estadística puede dar una idea de la importancia de tal dedicación y esfuerzo. No toda esta producción la constituyen tratados científicos propiamente dichos. Junto a estos, abundan también los aforismos, las recensiones de obras de carácter científico, las anotaciones puntuales y las observaciones autobiográficas, encontrándose los textos unas veces muy bien elaborados y otras meramente esbozados.

Entre los escritos científico-naturales de Goethe, los que ocupan una mayor parte son los trabajos sobre la teoría de los colores. Junto a estos hay una serie de trabajos y bosquejos publicados sobre Zoología, Botánica, Mineralogía y Climatología.

2. Gedenkausgabe der Werke, Briefe und Gespräche, ed. Ernest Beutler, Artemis Verlag, Zürich, 1948-1954. Esta edición suiza se reparte en 24 volúmenes. Los números 16 y 17 se titulan Zur Naturwissenschaft y fueron editados bajo la supervisión del profesor Hans Fischer.

3. Werke, Kommentare und Register. Hamburger Ausgabe, editada y comentada por Erich Trunz, Christian Wegner Verlag, Hamburg, 1955-1971. Es la llamada Edición de Hamburgo. Está constituida por 14 tomos. Los dos últimos, los Naturwissenschaftliche Schriften, han estado al cuidado de Dorothea Kuhn ${ }^{13}$ y Rike Wankmüller.

4. Goethes Sämtliche Werke, Jubiläums-Ausgabe; Stuttgart y Berlín: J. G. Cotta`sche Buchhandlung Nachfolger ${ }^{14}, 1902-1907$. Comprende 40 volúmenes. Ésta ha sido una de las ediciones con más éxito; de hecho, hasta los años setenta se continuaron reeditando versiones completas y obras aisladas.

5. Goethes Werke, edición completa en 40 tomos. Auf Grund der Hempelschen Ausgabe neu herausgegeben, Bong\&Co., Berlin, Leipzig, Wien, Stuttgart, 1909. Los cua-

\footnotetext{
${ }^{12}$ En realidad el último volumen constituye un registro de cosas y personas.

${ }^{13}$ En las próximas líneas rescataremos la figura de esta gran científica y erudita que durante tantos años ha trabajado los escritos de carácter científico de Goethe para la edición Leopoldina.

${ }^{14}$ Es la editorial de los sucesores de Johann Friedrich Cotta (1764-1832), el que fuera el primer editor de las obras de Goethe y de otros clásicos de la literatura alemana.
} 
tro últimos volúmenes (36-40), Zur Naturwissenschaft, han estado al cuidado de Salomon Kalischer $^{15}$.

6. Die Schriften zur Naturwissenschaft, Vollständige mit Erläuterungen versehene Ausgabe herausgegeben im Auftrag der Deutschen Akademie der Naturforscher Leopoldina, ed. R. Matthaei, W. Troll und K. Lothar Wolf, Böhlau, Weimar, año 1947 y siguientes. Esta edición se conoce como la Leopoldina Ausgabe (LA) o edición leopoldina y en ella nos detendremos algo más, ya que se trata de una edición más moderna y explicada con detalle sobre las obras de carácter científico de Goethe; la primera parte se publicó en 11 tomos comentados por encargo de la Deutschen Akademie der Naturforscher Leopoldina -Academia alemana de científicos Leopoldina- entre 1947 y 1970. La segunda parte se empezó a editar en 1959 y hasta la fecha existen 16 volúmenes publicados. Aquí se han reunido todos los textos publicados y no publicados, provistos de comentarios muy completos, materiales y testimonios de otras obras y cartas que aclaran y explican el origen y el fondo de la obra de este genial autor.

Antes de contar brevemente la historia de esta edición hay que tener en cuenta que Goethe fue miembro de esta Academia. La Academia Leopoldina fue fundada en 1652 por cuatro médicos bajo el nombre de Academia Naturae Curiosorum con el objetivo de investigar en el campo de la medicina y servir de provecho para los demás. Ésta se concibió desde un primer momento como una asociación de eruditos de carácter internacional. En $1670^{16}$ apareció el primer ejemplar de la revista fundada por la Academia a la que se considera como la primera serie de publicaciones de carácter científico-médico editadas sin interrupción. Este primer volumen estuvo dedicado al emperador Leopoldo $\mathrm{I}^{17}$ y de ahí su nombre de Academia Leopoldina. Posteriormente se ampliarían los objetivos científicos de la academia diversificándolos en distintas áreas de conocimiento (cf. Ter Meulen -ed-, 2009: 7-9).

Como hemos comentado con anterioridad, los estudios de carácter científico ocuparon gran parte de la vida y obra de Goethe; sin embargo, aunque la influencia de estos estudios en su obrar y pensar encontraron su expresión artística fundamentalmente en la poesía, sus coetáneos no valorarían de igual forma su faceta poética y científica. Como explica Wieland Berg (1991: 109-126), muchos especialistas de su época vieron en Goethe el amateur que arremete contra los principios establecidos con ideas insuficientemente fundamentadas. Tan sólo supieron apreciar los esfuerzos y trabajos de Goethe algunos

\footnotetext{
${ }^{15}$ Salomon Kalischer (1845-1924), nació en la antigua Prusia y estudió en el seminario judío de Breslau. Posteriormente estudió en la Universidad de Berlín física y química, llegando a obtener un puesto docente en la Escuela Técnica Superior de Charlottenburg. Editó las obras de carácter científico de Goethe con notas e introducción, editadas originalmente en los años 1877-79 por G. Hempel, vols. XXXIII-XXXVI. También realizó el capítulo «Goethe als Naturforscher» para la biografía que escribió sobre Goethe Albert Bielschowsky (cf. 1904: 412-461).

${ }^{16}$ Fundada por el físico Sachs von Lewenhaimb, esta publicación de carácter científico lleva el barroco título de «Miscellanea curiosa medico-physica Academiae Naturae Curiosorum sive Ephemeridum medico-physicarum germanicarum curiosarum annus Primus».

${ }^{17}$ Leopoldo I de Habsburgo (1640-1705). Emperador del Sacro Imperio Romano Germánico desde 1658. Nieto del rey de España Felipe III por vía materna.
} 
representantes de la joven generación ya existente de naturalistas bajo la influencia de la Naturphilosophie o Filosofía natural alemana. Uno de ellos fue el botánico Christian Gottfried Daniel Nees von Esenbeck ${ }^{18}$ (1776-1858), presidente de la Leopoldina desde 1818; de hecho, durante su cargo como presidente, Goethe fue uno de los primeros en ser elegido miembro de la Academia siendo su sobrenombre Arion IV. También los dos presidentes posteriores tendrían una estrecha relación con Goethe. Dietrich Georg Kieser -presidente de 1858 a 1862- fue profesor de medicina en Jena desde 1812 y estuvo en numerosas ocasiones convidado en casa de Goethe. Con el médico y pintor Carl Gustav Carus -presidente de 1862 a 1869- mantuvo Goethe correspondencia epistolar sobre cuestiones de carácter científico y artístico. En la obra que publicó Carus en 1843, Goethe. Zu dessen näherem Verständnis, hizo una caracterización de lo que supusieron las investigaciones científicas de Goethe en el conjunto de sus obras.

La idea de publicar una edición exclusivamente con las obras de carácter científico de Goethe fue del fisicoquímico Karl Lothar Wolf ${ }^{19}$ (cf. Wolf, K. L., 1957) y de la estudiante de filología clásica y posteriormente editora, Leiva Petersen ${ }^{20}$. Esto ocurrió en los años treinta al observar que en las ciencias naturales de su tiempo se estaba produciendo una orientación de marcado carácter positivista que querían confrontar dando a conocer el método de proceder morfológico global de Goethe. Para poder llevar esto a cabo se tenía que poner al alcance del público las obras de carácter científico de Goethe de forma asequible y con un formato adecuado. La historia de su publicación la cuentan los propios editores (cf. Grumach, E. \& Wolf, K.L.,1958) y aparece también de forma resumida en la página web de la Academia Leopoldina (cf. http://www.leopoldina-halle.de/goethe/gesch.htm\#ausgabe) . En 1937, Wolf acepta una cátedra en Halle, donde coincide con el botánico Wilhelm Troll ${ }^{21}$, quien en 1926 había publicado una selección de trabajos morfológicos de Goethe; incluyeron también a Günther Schmid, investigador sobre Goethe, botánico y profesor de Historia de las ciencias naturales cuya recopilación bibliográfica Goethe und die Naturwissens-

\footnotetext{
${ }^{18}$ Christian Gottfried Daniel Nees von Esenbeck (1776-1858). Médico y botánico. Inició su carrera profesional como médico; después conseguiría la cátedra como profesor de botánica y filosofía de la naturaleza primero en Erlangen (1818), después en Bonn (1819), Breslau (1831) y Berlín (1848). Visitó a Goethe en Weimar en 1816 y 1819 ; de este encuentro surgiría un intercambio epistolar entre ambos que duraría muchos años. Uno de los libros que Nees escribió en 1816 sobre hongos y setas lo estudiaría Goethe al detalle. Nees se encargaría junto con el botánico Karl Friedrich Philip von Martius (1794-1868) de poner el nombre en honor a Goethe a una malva procedente de las selvas brasilianas: Malva Goetheae (cf. Bohley, J., 2003).

${ }^{19}$ Karl Lothar Wolf (1901-1969), docente e investigador alemán de gran prestigio. Desarrolló su trabajo en varias universidades alemanas. En 1930 se le nombra catedrático de Química Física de la Universidad de Kiel, donde compaginó su actividad investigadora con el cargo de rector de esta universidad entre 1933 y 1935.(cf. UAH PA 17240 Wolf, Rep. 6 Nr. 1407, Leopoldina-Archiv MM 4538 Wolf en: www. catalugusproffesorumhalensis.de ).

${ }^{20}$ Leiva Konstanze Petersen (1912-1992). Relevante personalidad científica de la extinta RDA.

${ }^{21}$ Wilhelm Troll (1897-1978). Bávaro, licenciado en Botánica por la Universidad de Múnich. En 1932 consigue una plaza de profesor en la Universidad de Halle, desde donde dirige el instituto y el jardín botánico de esa universidad. En 1945 fue deportado a zona de ocupación francesa, pudiendo ejercer desde 1946 sus labores como docente en la Universidad de Maguncia. La excelente biografía que sobre él realizó Gisela Nickel proporciona innumerables datos (cf. Nickel, 1996), así como el catálogo de profesores de la universidad de Halle (cf. www.catalugusproffesorumhalensis.de), que ha elaborado el Archivo de esta Universidad.
} 
chaften (Goethe y las ciencias naturales) ${ }^{22}$ acababa de publicarse en 1940. Wolf, Troll y Schmid eran miembros de la Academia Leopoldina; y así, con el apoyo de Leiva Petersen, que entre tanto había conseguido empezar a trabajar en la dirección de la conocida editorial de Weimar Hermann Böhlau Nachfolger -la editorial que años antes publicó las obras completas de Goethe, más conocida como la Sophie Ausgabe-, consiguieron también el apoyo para su plan de Emil Abderhalden ${ }^{23}$, presidente de la Academia, para editar las obras científicas de Goethe. Abderhalden, que de todos modos estaba interesado en trabajos de carácter científico-histórico, no dudó en ponerse en contacto con su predecesor en el cargo, Johannes Walter, quien en 1930 había publicado un volumen en memoria de Goethe como naturalista y miembro de la Academia Leopoldina.

En mayo de 1941 Abderhalden encarga a Schmid, Troll y Wolf la edición de las obras científicas de Goethe, pudiendo firmar unas semanas más tarde el contrato con la editorial de Weimar Hermann Böhlaus Nachfolger, con la que mantenían una relación desde hacía décadas. De esta forma, y tras haber zanjado un conflicto que mantenían desde principios de los años treinta sobre la edición de las obras completas de Goethe, pudieron comenzar los editores su labor. Sin embargo, la II Guerra Mundial y sus consecuencias pronto se hicieron notar en la edición; así, Troll y Wolf se vieron obligados a abandonar Halle el 24 de junio de $1945^{24}$ y vivieron desde entonces en la zona de ocupación francesa, es decir, lo que después sería el Land (estado federado) de Renania Palatinado. Dificultades de carácter técnico retrasaron la aparición de primer volumen que hasta 1947 había estado al cargo de Günther Schmid; el segundo volumen aparecería en el año en que falleció, en 1949. Al suspenderse la publicación de las obras completas de Goethe, el fisiólogo Rupprecht Matthaei ${ }^{25}$ ofreció su colaboración a la Academia Leopoldina para la investigación de los estudios de óptica y la teoría de los colores de Goethe hasta 1808. De esta forma se le admitió entre los editores y su volumen apareció en 1951.

${ }^{22}$ Günther Schmid (1888-1949). Desde 1935 trabajó en el Instituto botánico de la Universidad de Halle. Tan sólo 3 años antes de su fallecimiento sería reconocida su labor científica con una plaza como profesor de Historia de las Ciencias Naturales y Farmacognosia. Ayudó a la desnazificación de su universidad al terminar la II Guerra Mundial (cf. Schmid, G. 1940). Sobre su biografía cf. www.catalugusproffesorumhalensis.de

${ }^{23}$ Emil Abderhalden, (1877-1950). Oriundo del cantón suizo de Sankt Gallen. Estudió medicina en la Universidad de Basilea, doctorándose en 1902. De esta forma pudo entrar a trabajar 2 años más tarde en los laboratorios del que fuera premio Nobel Emil Fischer. En 1911 obtuvo una plaza de profesor en la Facultad de Medicina de la Universidad de Halle, donde permanecería hasta finalizar la II Guerra Mundial, a pesar de las numerosas invitaciones de otras universidad para dar clase en sus aulas. Su autobiografía queda reflejada en un artículo que escribe en 1938 titulado «Zum Abschied» (cf. Abderhalden, 1938: 3-31).

${ }^{24}$ Unos días antes de que los americanos, que habían ocupado Halle, entregaran la ciudad a la administración soviética, numerosos científicos y técnicos de la universidad, así como de las empresas químicas circundantes y de los talleres de aviación, se vieron obligados a abandonar la ciudad junto con sus familias y colaboradores. Eran algo más de 700 las personas que fueron forzadas a montarse en los vagones erróneamente conocidos como «Abderhaden-Transport»-siguiendo el apellido del bioquímico Emil Abderhalden quien en ese momento dirigía la Academia Leopoldina- con destino a Darmstadt, en el Oeste de Alemania. Una vez allí no tuvieron ninguna posibilidad ni de retorno ni de trabajo (cf. Kleinert, A, 2003: 140).

${ }^{25}$ Rupprecht Matthaei, nacido en Kiel en 1875. Licenciado en medicina y catedrático de fisiología desde 1935 en la Universidad de Tubinga y posteriormente en Erlangen. Científico comprometido con el régimen nacionalsocialista (cf. Grüttner, M. 2004). Falleció en Erlangen el 6 de enero de 1976 (cf. UAT 257 en www. www.catalugusproffesorumhalensis.de). 
Después se tendrían en cuenta las modificaciones personales y nuevas exigencias, de tal forma que se revisó la concepción de la edición leopoldina en su totalidad.

A comienzos de los años cincuenta, Karl Lothar Wolf se puso en contacto con Ernst Grumach -editor de una de las ediciones crítico históricas de las obras de Goethe que la Academia de Berlín estaba preparando-, así como con el editor de los escritos de carácter administrativo de Goethe, Willy Flach ${ }^{26}$. De esta forma surgió el plan de reconvertir la edición Leopoldina en una edición crítico histórica, unificándose criterios editoriales y formales para poder utilizar las tres ediciones como serie de una nueva edición de las obras completas de Goethe. En 1958 apareció la primera edición tras haber acordado los principios editoriales comunes. El nuevo concepto se pudo realizar fundamentalmente gracias a la bióloga y germanista Dorothea Kuhn ${ }^{27}$ (cf. Kuhn, D. 1992 y 1999), quien desde 1952 trabajaba en la edición de la leopoldina y desde 1954 formaba parte del equipo editorial, así como al mineralogista Wolf von Engelhardt, quien, tras muchos años como colaborador, pasó a engrosar las filas del equipo editorial en 1970. Hay que agradecer especialmente a Dorothea Kuhn que este gran proyecto editorial no hubiera fracasado como consecuencia de la división de Alemania al término de la II Guerra Mundial. Tras el fallecimiento en 1969 de Karl Lothar Wolf, en 1976 de Rupprecht Matthaei y en 1978 de Wilhelm Troll, los responsables de la publicación de la edición Leopoldina fueron Dorothea Kuhn y Wolf von Engelhardt. En 1976 consiguieron que el físico e historiador de la ciencia Horst Zehe se adhiriera al equipo que trabajaba en la edición de los escritos de Goethe sobre Óptica y Cromática.

Tras la reunificación de Alemania se consiguió a través del presidente de la Academia -Benno Parthier- y de los editores la institucionalización de la edición leopoldina, tan añorada desde sus comienzos. Se consiguieron además dos puestos más para la plantilla de la Academia financiados por el Land de Sajonia-Anhalt y la Comisión Bund-Länder-Kommission, de forma que pudieron contratar a la bióloga e historiadora de la ciencia Gisela Nickel, que en estos momentos trabaja en el segundo volumen de la segunda parte y al dentista e historiador de la medicina Thomas Nickol quienes, junto con Horst Zehe, continúan trabajando en la edición de los escritos de Goethe sobre cromática a partir de 1810. Desde 1996 la responsable de la edición es la comisión de la Academia Leopoldina Wissenschaftliche Akademievorhaben o «Proyectos científicos de la Academia».

${ }^{26}$ FLACH, W.; DAHL, H., Goethes amtliche Schriften. Veröffentlichung des Thüringischen Hauptstaatsarchives Weimar. Bd. 1-4. Weimar 1950-1987.

${ }^{27}$ Dorothea Kuhn nace en Halle an der Saale el 11 de marzo de 1923. Mujer de un tesón asombroso, que la lleva a continuar sus estudios de biología tras la II Guerra Mundial y tras haber sido expulsada de Halle a Darmstadt en el mal llamado «Abderhalden-Transport». Se doctoró en 1952 por la Universidad de Maguncia, siendo su director de tesis Wilhelm Troll. Su tesis doctoral se centraba en un problema de morfología de las plantas, lo que constituiría su punto de encuentro con Goethe. En su currículum cabe destacar su habilitación por diferentes universidades como las de Tubinga (1970) y Heidelberg (1977), donde llegó a ser catedrática. Asimismo, dirigió el Archivo de Cotta. Sin embargo, en lo que ha desplegado sus energías con gran intensidad, ha sido en la edición leopoldina de las obras de Goethe. Realmente y citando a Benno Parthier «ella ha sido la que nos ha enseñado que no se puede entender ni juzgar al poeta y pensador Goethe sin el conocimiento de Goethe como investigador de la naturaleza» (cf. Kleinert, A., 2003). 


\section{LA REPERCUSIÓN DE LA PRODUCCIÓN CIENTÍFICA DE GOETHE}

Como podemos observar después de haber detallado la historia de la edición leopoldina de las obras de Goethe, ésta tiene gran importancia por su esmero y por la valoración tan positiva que hace de la faceta científica del escritor. Hemos comentado con anterioridad que ya en vida de Goethe sus obras de carácter científico tuvieron escasa repercusión y fue valorado injustamente. Sin embargo, cabe destacar la gran acogida que tuvo Goethe entre los miembros de la Academia Leopoldina. Nos encontramos, pues, ante un problema en cuanto a la resonancia o recepción de sus obras. Son muchos los factores que han llevado a que la producción científica de Goethe haya tenido escasa resonancia y se haya contemplado ésta con relativa indiferencia. Uno de los más importantes ha sido la poco afortunada historia de sus interpretaciones dominada por la incomprensión y la unilateralidad que -como explica Sánchez Meca (1997: XII)- ha logrado mantener alojadas durante mucho tiempo las ideas de Goethe en ese margen de las antiguallas «idealistas», metafísicas, precientíficas que bien merecen seguir cubriéndose de polvo en los museos y en las bibliotecas. De hecho, el mismo Goethe tuvo que pelear en vida contra la incomprensión general de sus contemporáneos, como cuenta en muchas ocasiones en sus escritos, en los que se queja de la obstinación de sus colegas y la envidia e incomprensión por parte de amigos y editores. En su obra El autor expone la historia de sus estudios botánicos nos lo deja bien claro:

(...) desde hace más de medio siglo, en mi país y sin duda también fuera de él, se me conoce como poeta, o en todo caso se me permite pasar por tal; pero no es de tan general conocimiento ni ha suscitado atención el hecho de que me he dedicado con asiduidad y gran atención a la Naturaleza en sus fenómenos físicos generales y orgánicos, y que siempre he seguido en silencio y con pasión las observaciones serias de la misma. Por esta razón, pues, al publicarse hace cuarenta años en lengua alemana ese ensayo mío, en el que se trata de representar ingeniosamente las leyes de la morfología vegetal, y divulgarse ahora más, sobre todo en Suiza y Francia, no salía la gente de su asombro de que un poeta, que, por lo general, sólo entendiera en fenómenos morales, pertinentes al mundo del sentimiento y la imaginación (...) hubiera podido acertar de pasada con tan principal descubrimiento. Para salir al paso de este prejuicio se ha escrito, en realidad, el artículo que antecede; el cual está llamado a poner de resalto cómo he tenido yo ocasión de consagrar gran parte de mi vida, llevado de afición fervorosa, a los estudios naturales. Así que, no por ningún dote extraordinario del espíritu, ni debido a ninguna momentánea inspiración, sino en virtud de un continuo esfuerzo, he podido llegar finalmente a resultado tan satisfactorio ${ }^{28}$.

También Sánchez Meca (1997: XIII) corrobora estas ideas al afirmar que «Nietzsche, a no muchos años de distancia todavía de Goethe, constataba cómo, de las mejores ideas de su pensamiento científico, no permanecía vivo ya prácticamente nada».

Otro punto que debe tenerse en cuenta dentro de la escasa resonancia de su producción científica es que -como consecuencia del poco valor atribuido a su faceta científica-

${ }^{28}$ cf. J. W. v. Goethe, El autor expone la historia de sus estudios botánicos. Tomo II, pág. 1995. Traducido para la Editorial Aguilar en 1950 por Rafael Cansinos Assens. 
los no germano parlantes han tenido pocas oportunidades de conocer de primera mano los pensamientos de Goethe en lo referente a los contenidos de carácter científico. Durante mucho tiempo, la principal fuente en inglés de sus escritos sobre el método científico fue la selección realizada por Bertha Mueller en Los escritos botánicos de Goethe ${ }^{29}$. Este volumen contiene traducciones de todos los ensayos importantes de Goethe sobre metodología científica y botánica; sin embargo, nunca fue reeditado y durante décadas era prácticamente imposible encontrarlo. La mayoría de estos ensayos, junto con algunos ensayos de Goethe que no se habían traducido antes, volvieron a traducirse posteriormente en la obra de Douglas Miller Goethe: estudios científi$\cos ^{30}$. Jeremy Naydler (1996: 39-40) explica que, aparte de estas dos importantes antologías de escritos científicos de Goethe, ha habido numerosos intentos de mostrar, de manera más o menos sistemática, su punto de vista. Según este autor la tentativa de mayor éxito en los últimos años es sin duda La conciencia científica de Goethe, de Henri Bortoft ${ }^{31}$.

En el mundo hispanohablante tenemos traducidas muchas obras literarias de Goethe. Las Obras Completas traducidas por Rafael Cansinos Assens y publicadas por la editorial Aguilar (1987) ${ }^{32}$ tuvieron una gran repercusión, aunque ésta no sería la primera traducción ya que con anterioridad se habían realizado otras dos versiones españolas: la de J. F. Matheu en 1867 y la de Ramón María Tenreiro en 1929; ésta última, fácilmente asequible en la Colección Austral ( ${ }^{\circ}$ 752) de Espasa-Calpe. No obstante, aunque se hable de Obras Completas, esto no significa que incluyan todas las obras de Goethe sino solamente las clásicas, es decir, las más conocidas y relevantes dentro del ámbito literario. Así pues, dar con las obras de carácter científico de nuestro autor en lengua española no es labor sencilla. Ciertamente, en la traducción de Cansinos Assens nos encontramos con una parte importante de los escritos científicos entre los que destacan: Esbozo de una teoría de los colores (Tomo I) y Particularidades autobiográficas (Tomo II), dentro de la que se encuentra en el capítulo V, Estudios y curso de cultura a) Para la «Teoría de los colores» b) Para la «Metamorfosis de las plantas», donde nos encontramos con el importantísimo ensayo autobiográfico de Goethe donde nos cuenta la historia de su dedicación a la Botánica «El autor expone la historia de sus estudios botánicos» c) Para la Zoología d) Para la Meteorología e) Para la Geología f) De arquitectura alemana.

El mismo traductor de la obra nos cuenta en la Advertencia al lector (vol. I: 7) de la segunda edición cómo han ampliado

\footnotetext{
${ }^{29}$ Se trata de la obra MUELLER, Bertha (1952): Goethe's Botanical Writings, University of Hawaii Press, Honolulu.

${ }^{30}$ La referencia completa es MILLER, Douglas (1988): Goethe: Scientific Studies, Suhrkamp, Nueva York; nueva edición (1995), Princenton University Press.

${ }^{31}$ BORTOFT, Henri (1972): Goethe's Scientific Consciousness, Institute of Germanic Studies, Londres. Reproducido en un estudio posterior más exhaustivo de Bortoft (1996): The Wholness of Nature: Goethe's Way of Science, Floris Book, Edinburgo; Lindisfarne, Nueva York.

${ }^{32}$ Las Obras Completas de Goethe fueron editadas en el año 1950 por la editorial Aguilar. Nosotros hemos utilizado la $4^{\text {a }}$ edición, $2^{\text {a }}$ reimpresión del año 1987. En el año 2003 la editorial Aguilar las volvió a editar en España; sin embargo, estas adolecen de la parte de contenido científico, así como parte del prólogo inicial de Rafael Cansinos Asséns.
} 
(...) el título de Obras literarias que llevaba la primera, y sustituídolo por este otro de Obras completas, no sólo por incluirse ahora en ella la Teoría de los colores, de carácter puramente científico, que rebasa el epígrafe de lo literario, sino también porque, gracias a esa inclusión, no queda fuera de nuestra recopilación goethiana ninguna de las obras que el escritor publicó en vida o dejó listas para su publicación póstuma (...) sólo echará de menos el lector poesías aisladas (...), simples notas bibliográficas (...), artículos sobre temas de ciencias naturales, de los que recogemos los más importantes, como los referentes a la morfología de las plantas y a su descubrimiento del hueso intermaxilar en el hombre, etc., etc. Y, desde luego, también sus numerosos Diarios y su no menos copioso Epistolario, que en realidad no pueden considerarse verdaderas obras de un autor, sino más bien datos para su biografía y documentos para la inducción de su psicología personal.

De este modo vemos que hay partes de la obra de Goethe de gran importancia para la comprensión de la vocación científica de este autor, en las que el registro utilizado en su traducción se ha quedado en parte algo obsoleto y, en ocasiones, falto de precisión en cuanto a los lenguajes específicos, como puede ser el de la Botánica, asunto comprensible si tenemos en cuenta la amplitud de áreas que trató Goethe. Asimismo, hay que señalar que no toda la producción de Goethe está actualmente disponible en lengua española; éste es el caso de sus cartas y diario que son de sumo interés para conocer no sólo su personalidad, sino también sus relaciones con personajes ilustres y desconocidos del ámbito de las ciencias naturales y, por ende, también del mundo científico en general. Por lo tanto, a la hora de trabajar con los pasajes en los que se cite a Goethe procedentes de su Diario y Epistolario, nos veremos obligados a traducirlos o resumir su contenido. A la hora de citar ambas obras, tendremos que indicar la fecha de la redacción tanto para las cartas como para el diario, ya que, al no disponer de estas fuentes en español, hemos considerado que es la manera más sencilla de localizar el pasaje citado en cualquier edición alemana que se maneje. Para la consulta de estas obras en alemán resulta muy práctica la versión electrónica de la llamada Digitale Bibliothek Band 10: Goethe: Briefe, Tagebücher, Gespräche, que se corresponde con la edición de Weimar. En cuanto a la obra de las Conversaciones, cuando no esté clara la referencia temporal, proponemos incluir la referencia completa de la obra. Otra de las obras de Goethe de carácter autobiográfico es la llamada Tag- und Jahresheften, más conocida como los Anales. Se trata de un resumen con el contenido de lo que Goethe consideraba había sido más importante cada año; a la hora de citar esta obra, sí que se tiene que hacer una referencia completa de la fuente para lograr una mayor precisión. La edición que proponemos también en este caso es la versión electrónica de la llamada Digitale Bibliothek Band 4: Goethes Werke que se corresponde con la conocida edición de Berlín o Berliner Ausgabe (Goethe-BA). Si la traducción ya existe, como sucede con algunas de sus obras, creemos entonces conveniente reflejar la traducción publicada.

Bien es verdad que han surgido recientemente otras publicaciones en español sobre la obra científica del autor como por ejemplo Teoría de la naturaleza ${ }^{33}$ que recoge en la primera parte los escritos de botánica publicados por Goethe en Naturwissenschaft

${ }^{33}$ Estudio preliminar, traducción y notas de Diego Sánchez Meca (1997). Editorial Tecnos. Madrid. 
überhaupt, besonders zur Morphologie. Ehrfahrung, Betrachtung, Folgerung durch Lebensereignisse verbunden así como Vorarbeiten zu einer Physiologie der Pflanzen (Trabajos preliminares a una fisiología de las plantas), Vorarbeiten zur Morphologie (Trabajos preliminares sobre la morfología) y Nacharbeiten und Sammlungen (Trabajos posteriores y recopilaciones). En la segunda parte se presenta una selección de los escritos gothianos más representativos sobre teoría de la naturaleza, contenidos en los volúmenes 11 y 13 de la $2^{\text {a }}$ sección de la edición de Weimar. Posteriormente se ha publicado también Goethe y la ciencia ${ }^{34}$. Estas dos últimas obras serán de lectura indispensable para el lector hispanohablante que quiera introducirse en los escritos de carácter científico de Goethe.

\section{REFERENCIAS BIBLIOGRÁFICAS}

Abderhalden, Emil (1938): «Zum Abschied». In: Ethik, 14. Jg., H. 6, Halle, S. 3-31.

BERG, Wieland (1991): «Arion IV. - Goethe als Mitglied der Leopoldina». In: Salve Academicum II.

Beiträge zur Geschichte der Deutschen Akademie der Naturforscher Leopoldina. Veröffentlichungen des Stadtarchivs Schweinfurt. S. 109-126.BIELSCHOWSKY, Albert (1904): Goethe, sein Leben und seine Werke, Munich, ed. C.H.Beck.BOHLEY, Johanna (2003): Christian Gottfried Daniel Nees von Esenbeck: ein Lebensbild. - Stuttgart, Wissenschaftl.

Bortoft, Henri (1972): Goethe's Scientific Consciousness, Londres, Institute of Germanic Studies. Reproducido en un estudio posterior más exhaustivo de Bortoft (1996): The Wholness of Nature: Goethe's Way of Science, Edinburgo, Floris Book; Nueva York, Lindisfarne.

ECKERMANN, Johann Peter (1836-1848): Gespräche mit Goethe in den letzten Jahren seines Lebens. Hrsg. Fritz Bergemann. Baden-Baden, Insel Verlag.

FLACH, Willy; DAHL, Helma (1950-1987): Goethes amtliche Schriften. Veröffentlichung des Thüringischen Hauptstaatsarchives Weimar. Bd. 1-4. Weimar.

GoETHE, Johann Wolfgang (1987): Obras Completas. $4^{a}$ edición, segunda reimpresión. Recopilación, traducción, estudio preliminar, preámbulos y notas a cargo de Rafael Cansinos Assens. Madrid, Ed. Aguilar.

- (1947): Die Schriften zur Naturwissenschaft (LA).Weimar, Verlag Hermann Böhlaus Nachf.

- (1902-1907): Goethes Sämtliche Werke, Jubiläums-Ausgabe; Stuttgart y Berlín, J. G. Cotta`sche Buchhandlung Nachfolger.

- (1887-1909): Goethes Werke. Goethes Briefe. Herausgegeben im Auftrag der Gro herzogin Sophie von Sachsen. Band 1-49, Weimar, Hermann Böhlau und H. Böhlaus Nachfolger Hachfolger.

- (1887-1919): Goethes Werke. Goethes Tagebücher. Herausgegeben im Auftrag der Gro herzogin Sophie von Sachsen. Band 1-15, Weimar, Hermann Böhlau und H. Böhlaus Nachfolger Hachfolger.

- (1948-1954): Gedenkausgabe der Werke, Briefe und Gespräche, Zürich, ed. Ernest Beutler, Artemis Verlag.

- (1997): Teoría de la naturaleza. Estudio preliminar, traducción y notas de Diego Sánchez Meca. Madrid, Editorial Tecnos.

\footnotetext{
${ }^{34}$ Se trata en realidad de una traducción de la obra inglesa de Goethe on Science. An anthology of Goethe's scientific writings de Jeremy Naydler (1996), Floris Books: La edición española la encontramos en la Editorial Siruela (2002), cuya traducción ha corrido a cargo de Carlos Fortea y Esther de Arpe.
} 
- (1999): Goethes Werke auf CD Rom . Digitale Bibliothek Band 4: Goethe (vgl. Goethe-Hamburger Ausgabe), Bethesda, Chadwyck-Healey Ltd.

- (1999): Goethes Briefe, Tagebücher, Gespräche auf CD Rom . Digitale Bibliothek Band 10. Bethesda, Chadwyck-Healey Ltd.

GRUMACH, Ernst \& WolF, Karl Lothar (1958): «Zu den Akademie-Ausgaben von Goethes Werken». En: Jahrbuch. der Goethe-Gesellschaft N.F. 20: 309-310.GRÜTTNER, Michael (2004): Biographisches Lexikon zur nationalsozialistischen Wissenschaftspolitik. Studien zur Wissenschafts- und Universitätsgeschichte, Bd. 6. Heidelberg, Synchron.KLEINERT, Andreas (2003), «Dorothea Kuhn zum 80. Gebutstag». In: Nachrichtenblatt der deutschen Gesellschaft für Geschichte der Medizin, Naturwissenschaft und Technik, S. 139-141

KuHN, Dorothea (1992): «Erfahrung, Betrachtung, Folgerung durch Lebensereignisse verbunden. Zur Geschichte der Leopoldina-Ausgabe von Goethes Schriften zur Naturwissenschaft». En: Zur Edition naturwissenschaftlicher Texte der Goethezeit. Acta Historica Leopoldina 20: 1120.

KuHN, Dorothea (1999): «Die Leopoldina-Ausgabe der Naturwissenschaftlichen Schriften Goethes». En: Jahrbuch 1999. Leopoldina (R3) 45 (2000): 315-330 .

Miller, Douglas (1988): Goethe: Scientific Studies, Nueva York, Suhrkamp; nueva edición (1995), Princenton University Press.

Mueller, Bertha (1952): Goethe's Botanical Writings, University of Hawaii Press, Honolulu.

NAYDLER, Jeremy (1996): Goethe y la ciencia. Prólogo de Henri Bortoft. Madrid, Editorial Siruela.

RUPPERT, Hans (1958): Goethes Bibliothek. Katalog. Herausgegeben von den Nationalen Forschungs- und Gedenkstätten der klassischen deutschen Literatur in Weimar (NFG). Weimar, Arion Verlag.

SCHMID, Günther (1940): Goethe und die Naturwissenschaften. Ed. Emil Abderhalden, Halle/Saale. Soto De Prado y Otero, Catalina C. (2009): Goethe y el bosque a través de sus textos, $1^{\mathrm{a}} \mathrm{ed} ., \mathrm{Va}-$ lladolid, Gallandbooks.

Ter Meulen, Volker (ed.) (2009): «Leopoldina: Geschichte, Struktur, Aufgaben». In: Deutsche Akademie der Naturforscher Leopoldina e.V. S. 7-9.

WAGNER, Maria (2007): Goethe und die Forstwirtschaft. Remagen - Oberwinter, Kessel Verlag. www.catalugusproffesorumhalensis.de Última consulta: 10.02.2010.

http://www.leopoldina-halle.de/goethe/gesch.htm\#ausgabe. Última consulta: 11.02.2010. 
